\title{
Wavefunction localization and its semiclassical description in a 3-dimensional system with mixed classical dynamics
}

\author{
M. Brack ${ }^{a}$, M. Sieber ${ }^{b}$ and S. M. Reimann ${ }^{c, a}$ \\ ${ }^{a}$ Institute for Theoretical Physics, Regensburg University, D-93040 Regensburg, Germany \\ ${ }^{b}$ Max-Planck-Institute for the Physics of Complex Systems, Nöthnitzer Str. 38, D-01187 Dresden, Germany \\ ${ }^{c}$ Lund Institute of Technology, P. O. Box 118, S-22100 Lund, Sweden
}

(November 3, 2018)

\begin{abstract}
We discuss the localization of wavefunctions along planes containing the shortest periodic orbits in a three-dimensional billiard system with axial symmetry. This model mimicks the self-consistent mean field of a heavy nucleus at deformations that occur characteristically during the fission process [1],2]. Many actinide nuclei become unstable against left-right asymmetric deformations, which results in asymmetric fragment mass distributions. Recently we have shown [3 - 4 , that the onset of this asymmetry can be explained in the semiclassical periodic orbit theory by a few short periodic orbits lying in planes perpendicular to the symmetry axis. Presently we show that these orbits are surrounded by small islands of stability in an otherwise chaotic phase space, and that the wavefunctions of the diabatic quantum states that are most sensitive to the left-right asymmetry have their extrema in the same planes. An EBK quantization of the classical motion near these planes reproduces the exact eigenenergies of the diabatic quantum states surprisingly well.
\end{abstract}

PACS numbers: 24.75.+i, 03.65.Sq, 21.10.Dr, 47.20.Ky

We have recently applied [3, 4] the periodic orbit theory [5] 6] to a three-dimensional cavity model with axially symmetric deformations that typically occur near the isomer minimum and the second maximum of the characteristic double-humped fission barrier [1.2] of many actinide nuclei. The boundary of the cavity in cylindrical coordinates $(z, \rho)$ is given by a shape function $\rho(z)$; the deformations are defined by an elongation parameter $c$ and an octupole-type left-right asymmetry parameter $\alpha$ (see Ref. [1] for the detailed definitions; the neck parameter $h$ is fixed to be zero in the present work). The shortest periodic orbits are found in planes perpendicular to the symmetry $(z)$ axis at locations $z_{i}$ given [6] by $\rho^{\prime}\left(z_{i}\right)=0$; they are just the shortest polygons (diameter, triangle, square, etc.) inscribed into the circular cross sections of the billiard system with radii $\rho\left(z_{i}\right)$. The oscillating part $\delta E$ of the total energy of the system containing $N$ fermions (we do not distinguish neutrons from protons and neglect the Coulomb and spin-orbit interactions) was calculated by the appropriate semiclassical trace formula. A uniform approximation was introduced to describe the bifurcation of a single orbit plane (at $\left.z_{0}=0\right)$ into three orbit planes $z_{i}(i=0,1,2)$ at the moment of the neck formation where the shape function $\rho(z)$ becomes convex (see Ref. [3] for details). The gross-shell features in $\delta E$ were emphasized by convolution of the trace formula over the wave number $k$ by a Gaussian of width $0.6 / R$ ( $R$ is the radius of the spherical nucleus), producing a smearing of the shell structure similar to that caused by the residual pairing interaction in realistic nuclear models [1]

In the quantum-mechanical calculations for the energy shell correction $\delta E$, derived from the quantum spectrum of realistic nuclear shell-model potentials using Strutinsky's shell-correction method [7], it was found that the outer fission barrier of many actinide nuclei is unstable against octupole-type deformations [8]. In our present parametrization, this barrier is located at $c=1.53$; by varying $\alpha$ from zero to $\sim 0.13$, the energy $\delta E$ is lowered by about $\sim 2.5-3 \mathrm{MeV}$. (For the present qualitative discussion, we neglect the smooth liquid-drop model part of the total energy which varies much less in this restricted region of deformations.) This result could be well reproduced in our semiclassical calculations [3, 1 . Th. Thereby, it
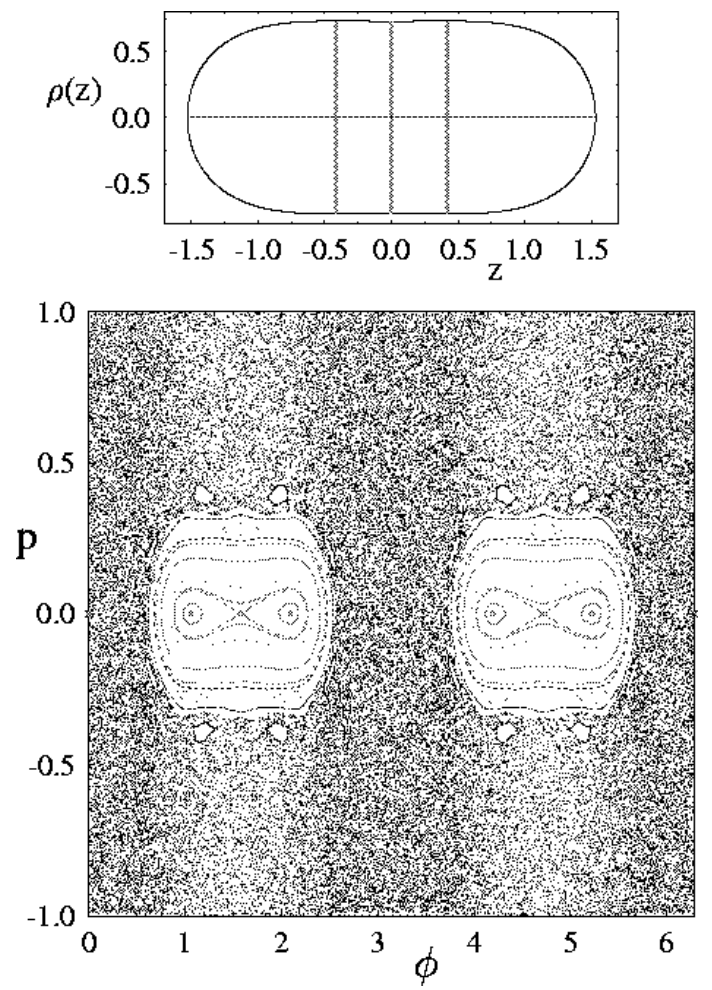

FIG. 1. Top: Cavity shape $\rho(z)$ at elongation $c=1.53$ and asymmetry $\alpha=0.0$. The vertical lines indicate the planes containing the shortest periodic orbits. Bottom: Poincaré surface of section $(p, \phi)$ for $L_{z}=0$. See the text for details. 
turned out to be sufficient to include the two shortest periodic orbits (diameters and triangles) in each of the planes $z_{i}$ into the trace formula; the contributions of longer orbits were shown to be negligible 何.

In the upper parts of Figs. 1 and 2, the shape boundaries $\rho(z)$ are shown for two deformations with fixed elongation $c=1.53$. The first corresponds to the symmetric outer barrier with $\alpha=0$, and the second to the asymmetric saddle with $\alpha=0.13$. The vertical lines show the positions $z_{i}$ of the planes containing the periodic orbits. In the symmetric case (Fig. 1), the shortest orbits at $z_{0}=0$ are slightly unstable, wheras those at $-z_{2}=z_{1}=0.414$ are stable. In the asymmetric case (Fig. 2), there is only one plane at $z_{0}=0.671$ containing stable orbits. In the lower parts of the figures, we show Poincaré surfaces of section $(p, \phi)$ for trajectories with (conserved) angular momentum component $L_{z}=0$, where $p$ is the component of the momentum parallel to the tangent plane at a reflection point and $\phi$ is the polar angle in the $(z, \rho)$ plane of a reflection point at the boundary. (Note that the Poincaré mapping in these variables is not area preserving; this is, however, immaterial for the qualitative interpretation of the figures.) In both cases we find two main islands of stability containing the fixed points corresponding to the shortest stable and unstable orbits mentioned above. Apart from some KAM chains of smaller islands corresponding to higher resonances, the remainder part of the phase space is mainly chaotic. Note that the overall chaoticity is much larger in the energetically
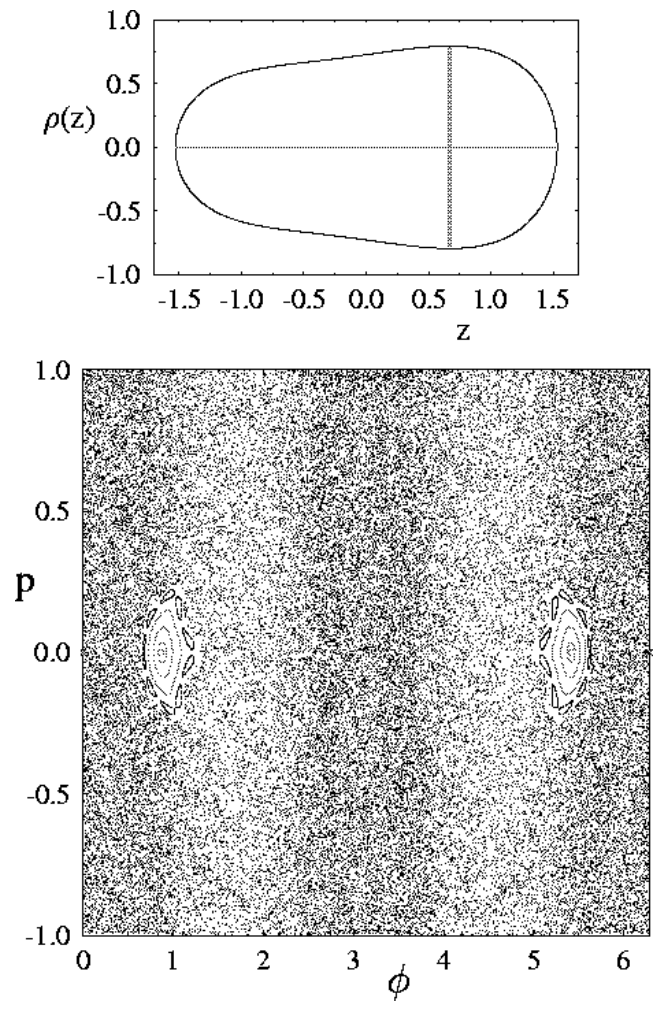

FIG. 2. The same as in Fig. 1 for asymmetry $\alpha=0.13$. more stable asymmetric case. We thus find that the shell effect which leads to the energetic instability of the symmetric outer barrier, at the same time pulls the system into a more chaotic transition state.

In Ref. [9], the microscopic origin of the instability against asymmetric deformations was linked to specific quantum states having their probability maxima in the central equatorial plane or in two planes parallel to it. These states were energetically most sensitive to the asymmetric deformations, whereas all others were practically not. In order to check this behaviour in our present cavity model and to investigate the relation of these states to the leading periodic orbits, we have calculated its quantum spectrum (with Dirichlet boundary conditions) at the relevant deformations. In Fig. 3 we show the wave number spectrum $k_{i}=\sqrt{2 m E_{i}} / \hbar$ (in units of $1 / R)$ for the lowest states $i$ with angular momentum $L_{z}=4$ obtained at $c=1.53$ versus the asymmetry parameter $\alpha$. Whereas most levels vary only little with $\alpha$, we clearly recognize a sequence of states whose energies decrease more and more steeply with increasing number $i$. These states are diabatic in that they connect different portions of the level spectrum through avoided crossings; in the upper part of the spectrum we have emphasized them by heavy grey lines and labeled them by the numbers $i$ of the adiabatic levels counted from the bottom. We shall show below that the energies of these diabatic states can be obtained from an EBK quantization of the classical motion near the equatorial planes.

The mechanism of the energy instability against $\alpha$ now

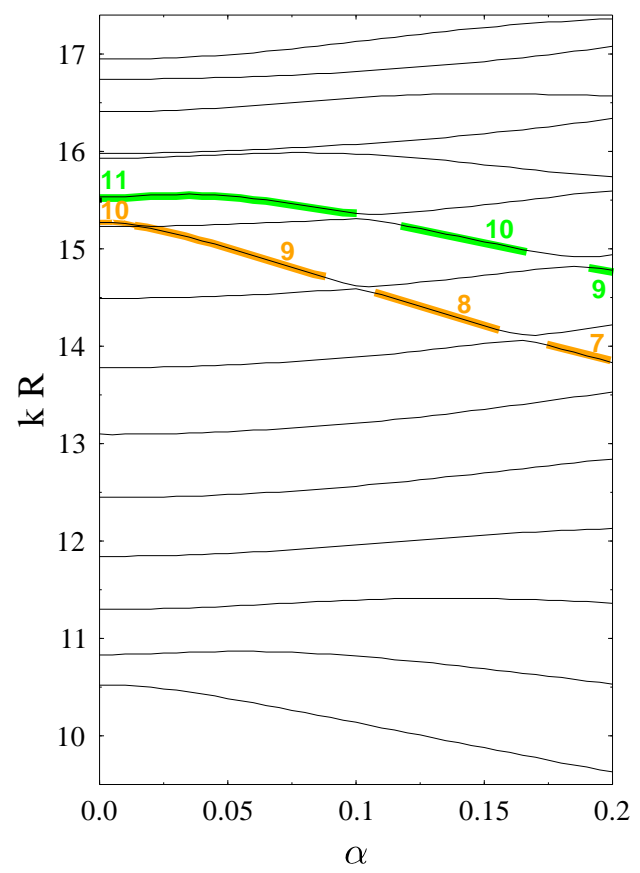

FIG. 3. Quantum levels $k_{i} R$ for the lowest states $i$ with $L_{z}=4$ at elongation $c=1.53$, plotted versus asymmetry $\alpha$. The emphasized portions connected by avoided crossings denote some of the diabatic states (see text). 
is this [9]: if one or two of the diabatic states are located just underneath the Fermi energy (and thus occupied), the system gains energy when $\alpha$ is increased from zero. Since all the occupied "inert" states slightly increase at larger values of $\alpha$, the sum of occupied levels (and hence $\delta E$, see Ref. [7]) will exhibit a minimum at some finite value $\alpha_{0}$. In our model calculation [3,4] for $\mathrm{Pu}^{240}$ with a fixed Fermi energy $k_{F}=12.1 / R$, we obtained $\alpha_{0} \simeq 0.13$.

In Fig. 4 we show probability distributions by contour plots of the squares of wave functions $\left|\psi_{i}(z, \rho)\right|^{2}$ in the $(z, \rho)$ plane. The centre and bottom panels correspond to $i=10$ at $\alpha=0$ and to $i=7$ at $\alpha=0.2$, respectively, representing one diabatic state at the two ends of the $\alpha$ interval. The top panel represents the beginning of the next higher diabatic state, starting as $i=11$ at $\alpha=0$. The probability maxima are clearly located precisely in the planes $z_{i}$ of the shortest periodic orbits, indicated by the heavy vertical lines. This pattern was found to be consistent: the probability maxima of all diabatic states are located in the planes of the shortest periodic orbits, whereas the maxima of all other "inert" states could not be correlated to any of the leading periodic orbits.

In order to quantify the correspondence between the diabatic quantum states and the leading periodic orbits, we apply a method analogous to the quasimode construc-
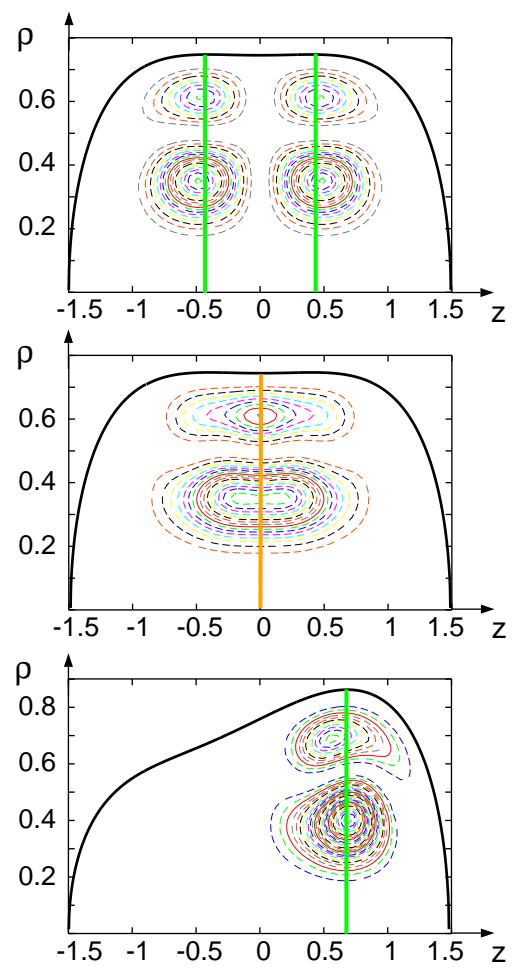

FIG. 4. Contour plots of the probability distributions $\left|\psi_{i}(z, \rho)\right|^{2}$ for selected states with $L_{z}=4$ at the elongation $c=1.53$. The heavy black lines show the shape $\rho(z)$ of the boundary. The grey vertical lines indicate the planes $z_{i}$ containing the shortest periodic orbits. Top: state $i=11$ at asymmetry $\alpha=0.0$. Centre: state $i=10$ at asymmetry $\alpha=0.0$. Bottom: state $i=7$ at asymmetry $\alpha=0.2$. tion for eigenfunctions located in the vicinity of stable periodic orbits (see, e.g., 10 ). Consider the classical motion in the vicinity of a stable equatorial plane. It consists of small oscillations around this plane. In a linearized approximation, the motion is integrable and restricted to tori in phase space. The quasimode approximation consists in applying the EBK quantization conditions to this torus structure [11]. To derive the quantization conditions, we use the fact that the quasimode approximation depends only on the two curvature radii at the boundary of the stable orbit plane: the radius $R_{1}$ in the equatorial plane itself, and the radius $R_{2}$ perpendicular to it. We therefore replace our billiard system locally by an axially symmetric ellipsoid with the same curvature radii of the equatorial planes. For an ellipsoid, the classical action variables are known [12]. In the vicinity of the equatorial plane they are given by the $z$ component of the angular momentum, $L_{z} / \hbar=0, \pm 1, \pm 2, \ldots$, by the action $I_{\rho}$ for the radial motion in the equatorial plane,

$$
\begin{aligned}
I_{\rho} \approx \frac{1}{\pi} & {\left[\sqrt{p^{2} R_{1}^{2}-L_{z}^{2}}-\left|L_{z}\right| \arccos \left|L_{z} /\left(p R_{1}\right)\right|\right.} \\
& \left.-\frac{\kappa}{2} \frac{\arcsin \sqrt{R_{1} / R_{2}-L_{z}^{2} /\left(p^{2} R_{1} R_{2}\right)}}{\sqrt{p^{2} R_{1}\left(R_{2}-R_{1}\right)+L_{z}^{2}}}\right],
\end{aligned}
$$

and by the action $I_{z}$ for the motion out of this plane,

$$
I_{z} \approx \kappa /\left[2 \sqrt{p^{2} R_{1}\left(R_{2}-R_{1}\right)+L_{z}^{2}}\right] .
$$

These actions depend on the three constants of motion: the energy $E$ (or the momentum squared $p^{2}=\hbar^{2} k^{2}$ ), the angular momentum $L_{z}$, and a conserved quantity $\kappa$ which vanishes for the motion in the equatorial plane and is the analogue of the second constant of motion in a two-dimensional elliptic billiard (given by the product of angular momenta with respect to the two foci). The actions (11), (2) are already given here in the linearized approximation obtained from the exact actions (given by elliptic integrals) through Taylor expansion up to first order in $\kappa$. Eliminating $\kappa$ from Eqs. (1), (2), we arrive at

$$
\begin{aligned}
I_{\rho}= & \frac{1}{\pi}\left[\sqrt{p^{2} R_{1}^{2}-L_{z}^{2}}-\left|L_{z}\right| \arccos \left|L_{z} /\left(p R_{1}\right)\right|\right. \\
& \left.-I_{z} \arcsin \sqrt{R_{1} / R_{2}-L_{z}^{2} /\left(p^{2} R_{1} R_{2}\right)}\right]
\end{aligned}
$$

to be used with the EBK quantization conditions

$$
\begin{aligned}
& I_{z}=\hbar(n-1 / 2), \quad n=1,2,3, \ldots \\
& I_{\rho}=\hbar(m-1 / 4) . \quad m=1,2,3, \ldots
\end{aligned}
$$

Note that if one puts $I_{z}=0$ above, Eqs. (3) and (5) yield precisely the radial EBK quantization condition (without further approximation) for a two-dimensional circular billiard with radius $R_{1}$.

Equations (3) - (5) represent our quasimode approximation. They implicitly yield the energies $E_{n m}$ (or wave 
numbers $k_{n m}$ ) of quantum states with angular momentum $L_{z}$ located near the planes of stable periodic orbits, which we expect to represent the diabatic states discussed above. The quantum numbers $n$ and $m$ count the wave function extrema in the $z$ and $\rho$ directions, respectively. (Correspondingly, $n-1$ and $m-1$ count the numbers of nodes in the respective directions.) E.g., the squared wavefunctions shown in the centre and bottom panels of Fig. 4 should represent the state $(1,2)$, and the one shown in the top panel should belong to the state $(2,2)$.

As a test of our interpretation of the diabatic quantum states and their semiclassical quantization, we compare in Fig. 5 the exact quantum spectrum $k_{i}$ (shown by solid lines) to the approximate EBK levels $k_{n m}$, calculated here for the lowest states with angular momentum $L_{z}=0$. Due to our linearization of the actions (11), (2), the agreement should be best for small values of the quantum number $n$. It is, indeed, perfect for all states with $n=1$ shown in Fig. 5 by the dotted lines, which agree exactly with the quantum levels of the corresponding diabatic states. The agreement is less good for the states with $n=2$ (shown by the dashed lines), but our semiclassical approximation still reproduces their correct slopes for larger $\alpha$ and allows for their unique assignment. Similar results are also obtained for larger values of $L_{z}$ (for which the numerical agreement actually improves).

In summary, we have established a correspondence between the shortest classical periodic orbits and a set of

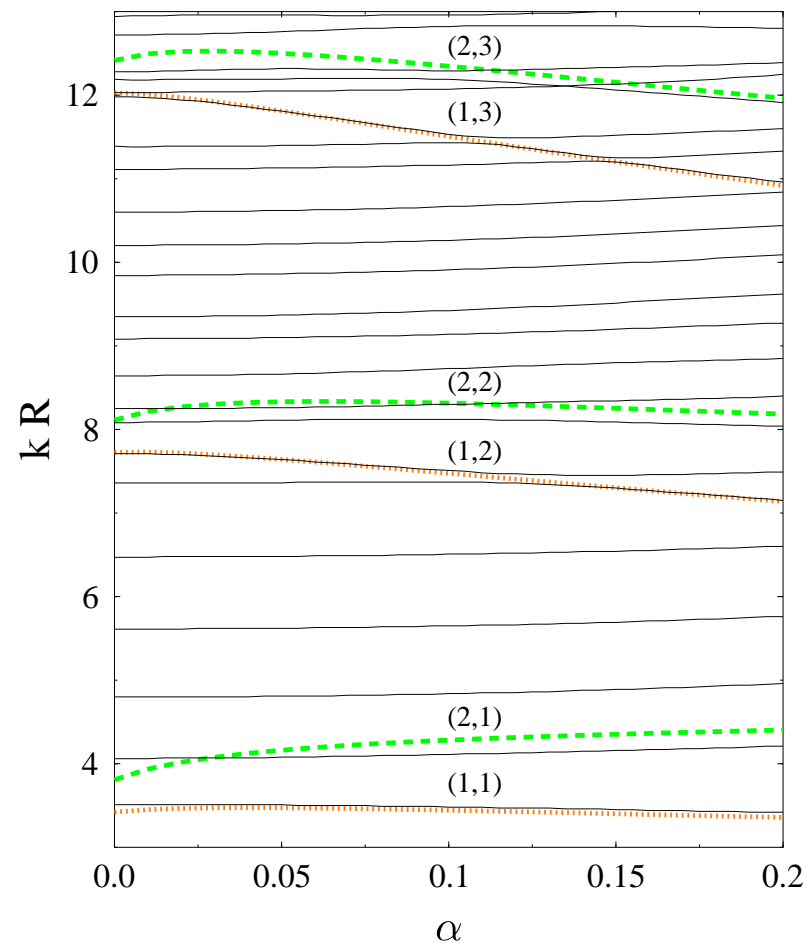

FIG. 5. Quantum levels $k_{i} R$ of lowest states $i$ with $L_{z}=0$ at elongation $c=1.53$ versus asymmetry $\alpha$ (solid lines). Labels $(n, m)$ indicate diabatic states; their levels $k_{n m} R$ obtained by the quasimode approximation, Eqs. (3) - (5), are shown by dotted lines for $n=1$ and dashed lines for $n=2$. diabatic quantum states responsible for the onset of the left-right asymmetry in a simple axially deformed cavity model describing schematically the shapes of an actinide nucleus on its adiabatic path to fission. The energy gain due to the asymmetric deformations near the outer barrier had earlier been reproduced by a semiclassical trace formula [3,4]; thereby the fission path through the deformation space was shown to be determined by the constancy of the actions of the shortest periodic orbits. In the present paper we have demonstrated that these orbits lie at the centres of small islands of stability in an otherwise chaotic phase space. Its degree of chaoticity is increased by the shell effect causing the asymmetric deformations. We have also shown that the diabatic quantum states which energetically favor the asymmetry have their probability maxima precisely in the planes where the shortest periodic orbits are located. A quasimode approximation based on EBK quantization of the linearized classical motion around the planes of the shortest stable orbits allowed us to uniquely assign quantum numbers $\left(L_{z}, n, m\right)$ to the diabatic quantum states and to semiclassically reproduce their energies rather well.

We acknowledge the help of $\mathrm{P}$. Meier in constructing the energy level plots. This work has been supported by the Deutsche Forschungsgemeinschaft (M.B.) and a Habilitationsstipendium des Freistaates Bayern (S.M.R.).

[1] M. Brack, J. Damgård, A. S. Jensen, H. C. Pauli, V. M. Strutinsky, and C. Y. Wong, Rev. Mod. Phys. 44, 320 (1972).

[2] S. Bjørnholm and J. E. Lynn, Rev. Mod. Phys. 52, 725 (1980).

[3] M. Brack, S. M. Reimann, and M. Sieber, Phys. Rev. Lett. 79, 1817 (1997).

[4] M. Brack, P. Meier, S. M. Reimann, and M. Sieber, in Similarities and Differences between Atomic Nuclei and Clusters, eds. Y. Abe et al. (AIP New York, 1998), p. 17.

[5] M. C. Gutzwiller, J. Math. Phys. 12, 343 (1971).

[6] R. Balian and C. Bloch, Ann. Phys. (N. Y.) 69, 76 (1972).

[7] V. M. Strutinsky, Nucl. Phys. A 95, 420 (1967); A 122, 1 (1968).

[8] P. Möller and S. G. Nilsson, Phys. Lett. 31 B, 283 (1970); H. C. Pauli, T. Ledergerber, and M. Brack, Phys. Lett. 34 B, 264 (1971).

[9] C. Gustafsson, P. Möller, and S. G. Nilsson, Phys. Lett. 34 B, 349 (1971).

[10] A. Voros, Colloques Internationaux du CNRS no. 237 (Aix-en-Provence, France, 24-28 June 1974), ed. J. M. Sourian (1975), p. 277.

[11] The quasimode treatment is limited to stable orbits. Note, however, that some wavefunctions are also found along planes of unstable orbits (see center of Fig. 4). This is similar to the scar phenomenon of the localization of wavefunctions near isolated unstable periodic orbits.

[12] P. H. Richter, A. Wittek, M. P. Kharlamov, and A. P. Kharlamov, Z. Naturforsch. 50a, 693 (1995). 